\title{
Marital Status in Moroccan Long-term Childhood Cancer
}

\section{Survivors}

\author{
Nadia Benaicha ${ }^{1}$, Fouzia Msefer Alaoui ${ }^{2}$, Karima Elrhazi ${ }^{1}$, Ousmane $\mathrm{SY}^{1}$, Ousmane Desire Diakité ${ }^{1}$ and Chakib \\ Nejjari ${ }^{1}$ \\ 1. Department of Epidemiology and Clinical Research, Faculty of Medicine, Fez, Morocco \\ 2. Department of Pediatric Oncology, Children Hospital of Rabat, Morocco
}

\begin{abstract}
In developed countries, currently, more than $80 \%$ of children with cancer survive and they are considered as cured. However, the survivors are at high risk of medical and social late effects. In Morocco, childhood cancer accounts for 3\% of all cancers. The purpose of this study was to look for factors related to marital status in Moroccan childhood cancer survivors. This was a cross-sectional study conducted among childhood cancer survivors treated between 1978 and 2004 at the Pediatric Oncology Unit of Children's Hospital of Rabat. Medical and demographic data were collected through questionnaires completed by phone or internet, by the survivor, his parent or his physician. Among 995 patients considered cured; only 195 were found. The decline from the initial diagnosis varies from 10 years to 36 years, with a mean of 16.2 years and a standard deviation of 6.3 years. In our study, we found that 31 survivors (22.3\%) of adult participants were married. They were aged between 23 and 43 (the mean was 31.8 and the SD was 5.9). Men, aged more than 35 years old and unemployed are less likely to be married. To our knowledge, there is no previous study about the fate of Moroccan childhood cancer survivors before our study. Hence, it was a cross-sectional study to have a quick picture on the situation in Morocco. It would be necessary to establish a follow-up strategy in Morocco.
\end{abstract}

Key words: Childhood, cancer, survivors, marriage, Morocco.

\section{Introduction}

Children's malignant tumors are major causes of death in children under 15 years old in the West [1]. According to data from literature, childhood cancers are dominated by leukemia, tumors of the central nervous system (CNS) tumors of the bone and soft tissue [2]. Young people cured of childhood cancer may develop complications related to the disease itself or the treatment. With advances in cancer treatment, today more than 80 percent of children diagnosed with cancer in developed countries are alive at least five years after diagnosis. Many ultimately will be considered cured [2]. However the survivors are at high risk of medical and social late effects. Complications can be somatic, psychological or socio-occupational. In 2007, Frobisher et al. reported

\footnotetext{
Corresponding author: Nadia Benaicha, research field: $\begin{array}{lll}\text { childhood cancer survivors. } & \end{array}$ nadia.benaicha79@gmail.com.
}

reduced marriage frequencies in 9,954 British childhood cancer survivors diagnosed from 1940 to 1991 compared to those expected from the general population and concluded that survivors were less likely to get married [3]. The British Childhood Cancer Survivor Study (BCCSS) which the database included 18.119 individuals, who were diagnosed with childhood cancer between 1940 and 1991, found that $66 \%$ of the 9.954 who were aged at least 16 years and eligible to receive a postal questionnaire had never been married. The category with the lowest probability of marriage were patients with CNS tumors, cranial radiation, impaired processing efficiency, and short stature. There is no difference in divorce patterns between survivors and their peers [4].

The objective of the study was to establish a long term follow up of survivors and identify medical and social disorders. The aim of this present survey was to describe marital status in childhood cancer survivors 
treated at pediatric oncology unit of the University Children Hospital of Rabat between 1978 and 2004 in order to evaluate social status.

\section{Materials and Methods}

\subsection{Type of Study}

This was a full coverage descriptive cross-sectional study.

\subsection{Study Population}

The study population consists of young people cured from childhood cancer ( 0 to 15 years) treated in Pediatric Oncology Unit of the Children Hospital of Rabat between 1978 and 2004. These young people were CR1 (complete remission without relapse) CR2 (complete remission after a single relapse) and CR3 (complete remission after 2 relapses).

This study has been presented to the local ethics committee of University Hospital Hassan II of Fez who agreed it. Informed consent has been sent to every potential participant. Questionnaires, upon receipt, have been assigned a unique number and at any time during the analysis of the study no person had been cited. These points were clearly stated in the informed consent so that the survivor of childhood cancer was not reluctant to give confidential information.

\subsection{Data Collection}

Initial and current medical and demographic data were collected through questionnaires completed by phone or internet by the survivor himself, his parent or his physician. Each subject enrolled in the study was contacted by phone or internet in order to present the purpose of the investigation and explain the necessity of participation of each subject. The revival was made for subjects who did not respond by telephone using social networks (facebook ...).

\subsection{Statistical Analysis}

All variables were summarized by using descriptive statistics. Categorical variables were described in terms of proportions and quantitative variables were described in terms of average extreme values and standard deviation.

The association between dependent variable (marital status) and several potential explanatory variables was investigated. Bi-variate analysis was used and the association between two categorical variables (for example marital status and sex) was searched by chi-square test, and was selected as the significance level a $P$ value of 0.05 .

Statistical analysis was done on SPSS Version 17.

\section{Results}

Among 995 patients considered cured by their last news, only 195 were found. Five (5) deaths from second malignancy or late relapse were included in our database.

\subsection{Descriptive Data}

The decline from the initial diagnosis varies from 10 years to 36 years, with a mean (an average) of 16.2 years and a standard deviation of 6.3 years. The male was dominant with Sex Ratio of 1.7. The range of ages of children varies from 1 month to 16 years, with a mean of 6.2 years and a standard deviation of 3.9 years. Ages 0-9 years are the most represented, totaling $77.8 \%$. More than $50 \%$ of the survivors completing questionnaires were diagnosed and treated during the period from 2000 to 2004 and $75 \%$ from 1995 to 2004 . The majority of our study population lived in urban areas $(77 \%)$.

The majority of the survivors had ALL (26.4\%), NHL (23.6\%), HD (14.8\%) and nephroblastoma (14.8\%). Almost all of the patients received chemotherapy, one third was operated on and one third irradiated. The vast majority of survivors never relapsed.

The majority of the participants were aged more than 18 years old $(71.6 \%)$ and 31 were married $(16.1 \%$ of adults). They were aged between 23 and 43 (the mean was 31.8 and the SD is 5.9). 19 (61.3\%) were 
employed and $9(37.5 \%)$ made sports (at least 3 hours per week).

\subsection{Correlations Marriage/Other variables}

The married survivors are more likely to be females $(P=0.048)$, to be less than 35 years old $(P<0.001)$, to be employed $(P=0.002)$ and to practice less than 3 hours sport per week $(P=0.042)$. The single survivors are more likely to live with their parents $(P<0.001)$. We didn't find relation statistically significant between marital status and education level $(P=0.143)$. No correlations have been found with the type of treatment received or the evolution of the disease (Table 2).

The married survivors have less physical problems than the not married (20.7\% VS $39.6 \%, P=0.045)$. We didn't find relation statically significant between marital status and aesthetic problems, cognitive problems, addiction or second cancer. We found a $\mathrm{p}$ value close to significance between marital status and psychical problems $(0.077)$ (Table 3$)$.

With the question of health subjective evaluation, we didn't find a difference between married and not married survivors $(P=0.269)$ (Table 4$)$.

Table 1 Descriptive data among 195 Moroccan childhood cancer survivors treated between 1978 and 2004.

\begin{tabular}{|c|c|c|}
\hline & Frequency & Percentage $(\%)$ \\
\hline \multicolumn{3}{|l|}{$\operatorname{Sex}(n=190)$} \\
\hline Female & 69 & 36.3 \\
\hline Male & 121 & 63.7 \\
\hline \multicolumn{3}{|c|}{ Adult $(\geq 18$ years old $)(n=194)$} \\
\hline Yes & 139 & 71.6 \\
\hline No & 55 & 28.4 \\
\hline \multicolumn{3}{|c|}{ Marital status $(n=139)$} \\
\hline Ever Married & 31 & 22.3 \\
\hline Not married & 108 & 77.7 \\
\hline \multicolumn{3}{|c|}{ Age at diagnosis in years $(n=189)$} \\
\hline $00-04$ & 81 & 42.9 \\
\hline 05-09 & 66 & 34.9 \\
\hline $10-15$ & 39 & 20.6 \\
\hline $15-19$ & 3 & 1.6 \\
\hline \multicolumn{3}{|c|}{ Years of diagnosis $(n=185)$} \\
\hline 1978-1984 & 7 & 3.8 \\
\hline 1984-1989 & 19 & 10.3 \\
\hline 1990-1994 & 20 & 10.8 \\
\hline 1995-1999 & 33 & 17.8 \\
\hline 2000-2004 & 106 & 57.3 \\
\hline \multicolumn{3}{|c|}{ Origin at diagnosis $(n=183)$} \\
\hline Urban & 142 & 77.6 \\
\hline Rural & 41 & 22.4 \\
\hline \multicolumn{3}{|l|}{ Cancers types } \\
\hline \multicolumn{3}{|l|}{ Treatment } \\
\hline Chemotherapy & 186 & 98.4 \\
\hline Radiotherapy & 64 & 34.8 \\
\hline Surgery & 70 & 37.4 \\
\hline \multicolumn{3}{|l|}{ Evolution } \\
\hline CRR & 148 & 93.0 \\
\hline $\mathrm{CR} 2$ & 9 & 5.7 \\
\hline CR3 & 2 & 1.3 \\
\hline
\end{tabular}


Table 2 Comparison of marital status with t socio-demographic data among 195 Moroccan childhood cancer survivors treated between 1978 and 2004.

\begin{tabular}{|c|c|c|c|}
\hline & Married & Not Married & $P$ value \\
\hline \multicolumn{4}{|l|}{$\operatorname{Sex}(n=137)$} \\
\hline Female & $16(51.6 \%)$ & $35(33.0 \%)$ & \multirow{2}{*}{0.048} \\
\hline Male & $15(48.4 \%)$ & $71(67.0 \%)$ & \\
\hline \multicolumn{4}{|l|}{ Current age $(n=136)$} \\
\hline $18-24$ & $6(20.0 \%)$ & $70(66.0 \%)$ & \multirow{5}{*}{$<0.001$} \\
\hline $25-29$ & $6(20.0 \%)$ & $23(21.7 \%)$ & \\
\hline $30-34$ & $9(30.0 \%)$ & $8(7.5 \%)$ & \\
\hline $35-39$ & $5(16.7 \%)$ & $4(3.8 \%)$ & \\
\hline $40-44$ & $4(13.3 \%)$ & $1(0.9 \%)$ & \\
\hline \multicolumn{4}{|l|}{ Education $(n=123)$} \\
\hline Not Educated & $1(3.3 \%)$ & $0(0.0 \%)$ & \multirow{5}{*}{0.143} \\
\hline Primary School & $4(13.3 \%)$ & $8(8.6 \%)$ & \\
\hline Middle School & $7(23.3 \%)$ & $15(16.1 \%)$ & \\
\hline High School & $3(10.0 \%)$ & $24(25.8 \%)$ & \\
\hline College/University & $15(50.0 \%)$ & $46(49.5 \%)$ & \\
\hline \multicolumn{4}{|l|}{ Habitat type $(n=130)$} \\
\hline With Parents & $5(16.7 \%)$ & $84(84.0 \%)$ & \multirow{3}{*}{$<0.001$} \\
\hline Alone & $0(0.0 \%)$ & $15(15.0 \%)$ & \\
\hline With partner & $25(83.3 \%)$ & $1(1.0 \%)$ & \\
\hline \multicolumn{4}{|l|}{ Working Status } \\
\hline$(\mathrm{n}=130)$ & & & \multirow{4}{*}{0.002} \\
\hline Employed & $19(61.3 \%)$ & $30(30.3 \%)$ & \\
\hline Unemployed & $8(25.8 \%)$ & $25(25.3 \%)$ & \\
\hline Student & $4(12.9 \%)$ & $44(44.4 \%)$ & \\
\hline \multicolumn{4}{|c|}{$\begin{array}{l}\text { Makes Sport (at least } 3 \text { hours per week) } \\
(n=109)\end{array}$} \\
\hline Yes & $9(37.5 \%)$ & $51(60.0 \%)$ & \multirow{2}{*}{0.042} \\
\hline No & $15(62.5 \%)$ & $34(40.0 \%)$ & \\
\hline
\end{tabular}

Table 3 Comparison of marital status with current medical data among 195 Moroccan childhood cancer survivors treated between 1978 and 2004.

\begin{tabular}{|c|c|c|c|}
\hline & Married & Not Married & $P$ value \\
\hline \multicolumn{4}{|c|}{ Physical Problems $(n=135)$} \\
\hline Yes & $6(20.7 \%)$ & $42(39.6 \%)$ & \multirow{2}{*}{0.045} \\
\hline No & $23(79.3 \%)$ & $64(60.4 \%)$ & \\
\hline \multicolumn{4}{|c|}{ Aesthetic Problems $(n=125)$} \\
\hline Yes & $2(7.4 \%)$ & $17(17.3 \%)$ & \multirow{2}{*}{0.166} \\
\hline No & $25(92.6 \%)$ & $81(82.7 \%)$ & \\
\hline \multicolumn{4}{|c|}{ Psychical Problems $(n=135)$} \\
\hline Yes & $3(10.3 \%)$ & $26(24.5 \%)$ & \multirow{2}{*}{0.077} \\
\hline No & $26(89.7 \%)$ & $80(75.5 \%)$ & \\
\hline \multicolumn{4}{|c|}{ Cognitive Problems $(n=61)$} \\
\hline Yes & $1(7.1 \%)$ & $6(12.8 \%)$ & \multirow{2}{*}{0.489} \\
\hline No & $13(92.9 \%)$ & $41(87.2 \%)$ & \\
\hline \multicolumn{4}{|c|}{ Addiction $(n=134)$} \\
\hline Yes & $2(6.9 \%)$ & $9(8.6 \%)$ & \multirow{2}{*}{0.561} \\
\hline No & $27(93.1 \%)$ & $96(91.4 \%)$ & \\
\hline \multicolumn{4}{|c|}{ Second Cancer $(n=135)$} \\
\hline Yes & $2(6.5 \%)$ & $1(1.0 \%)$ & \multirow{2}{*}{0.132} \\
\hline No & $29(93.5 \%)$ & $103(99.0 \%)$ & \\
\hline
\end{tabular}


Table 4 Comparison of marital status with subjective health evaluation among 195 Moroccan childhood cancer survivors treated between 1978 and $2004(n=162)$.

\begin{tabular}{lccc}
\hline & Married & Not Married & $P$ value \\
\hline Very good & $12(50.0 \%)$ & $52(37.7 \%)$ & \\
Good & $9(37.5 \%)$ & $38(27.5 \%)$ & 0.269 \\
Average & $3(12.5 \%)$ & $35(25.4 \%)$ & \\
Bad & $0(0.0 \%)$ & $10(7.2 \%)$ & \\
Very bad & $0(0.0 \%)$ & $3(2.2 \%)$ & \\
\hline
\end{tabular}

\section{Discussion}

To our knowledge, there is no previous study about the fate of Moroccan childhood cancer survivors before our study. In Morocco, treatment of childhood cancer is dominated by curative treatment. Hence, it was a cross-sectional study to have a quick picture on the situation in Morocco. The study had to be exhaustive, but only 195 among 995 were found. The survivors were diagnosed between 1978 and 2004 and the survey was made on 2014, which it means that we have decline from 34 to 10 years. Consequently, many of them have changed their coordinates. Furthermore, the questionnaire was elaborated to fill by the patients themselves, but for these who are absent, the filling was often made from medical records or their parents statements. However, retrieved data are interesting, and require reflection on many levels.

Saw Arab-Muslim context we only search legal married status, actually it can be considered as abasement to ask someone in Moroccan society about partner outside marriage. In our study "ever married" included only legally couple married or engaged.

Childhood Cancer Survivor Study found that adult female survivors of childhood cancer reported lower sexual functioning, lower sexual interest, lower sexual desire, lower sexual satisfaction and lower sexual activity compared with siblings [4]. By respect to Moroccan culture, we didn't have any question about sexuality.

Many studies had considered marriage as one of important indicators of psychological adjustment and success in social life [5-7]. Marriage can be considered as a positive outcome for the majority of young people in modern societies [8]. In our study we found that $83.9 \%$ of adult survivors (more than 18 years old) had never been married. British Childhood Cancer Survivor Study, which is a population-based cohort of 18,119 individuals who were diagnosed with childhood cancer between 1940 and 1991 and survived at least 5 years, found that $56 \%$ had never been married or lived as married [9]. The North American Childhood Cancer Survivor Study conducted among 25 oncology centers in USA and Canada and including self-reported data from 10.425 survivors who survived at least 5 years from diagnosis found that $62 \%$ having never been married [10].

In our study, we found $31(22.3 \%)$ married survivors among 136 adult participants (more than 18 years old) $[11,12]$. The identification of Moroccan population 2014 found that $57.3 \%$ men aged more than 15 years old and $57.8 \%$ women aged more than 15 years old are married [13]. Marriage can be considered as desirable outcome as most Moroccan adults aver 30 years old are married [13].

In our study, we found that men, aged more than 35 years old and unemployed are less likely to be married. In Moroccan context, having an income for a man is often important to be able to marry, because typically this is the man who must ensure the financial needs of the family. In this article we have not touched on fertility as we have little data among married survivors, but some male survivors reported psychological and social suffering related to their inability to have children, especially whom treated for Hodgkin Disease with more than 4 cures of MOPP.

Married survivors are less likely to practice sport, which can be explicated by the lack of sport culture in couple or family for many Moroccan people.

In our study we didn't find that marital status among 
survivors influence subjective health evaluation $(P=$ 0.269). But there is a tendency, none of married survivors has bad or very bad estimate of his/her health, but some of not married survivors consider their health as the same. Maybe if we increase the power of the study with more participant subjects, we can find a difference statistically significant.

\section{Conclusions}

The childhood cancer survivors are increasing in developed countries, but also in developing countries. However they stay at risk of late medical and demographic issues. Marriage is one of important indicators of social and psychological good health. The high rate of non-marriage found in our study shows that our survivors suffer from medical and social issues. It would be necessary to establish a long term follow-up strategy to improve the outcome of childhood cancer survivor in Morocco. This follow-up should provide medical care also psychosocial and educational support for patients and their families-

Author Contribution NADIA BENAICHA: Writing the protocol, drafting the questionnaire, writing the Consent Form, conducting the survey, Statistical Analysis, Writing the article FOUZIA MSEFER ALAOUI: Conception and writing the survey project, drafting and reviewing the questionnaires, reviewing the protocol, conducting and supervising the survey, reviewing the article KARIMA ELRHAZI: Reviewing the article OUSMANE SY: Statistical analysis OUSMANE DESIRE DIAKITE: Statistical analysis CHAKIB NEJJARI: Reviewing the protocol, reviewing the questionnaire, reviewing the Consent Form, reviewing the article.

\section{Competing Interests}

The authors declare that they have no competing interests.

\section{Funding}

This survey was funded by:
- The Avenir Association

- Terry Fox Foundation

\section{Acknowledgments}

Thanks to the team of Epidemiology of the University Hospital of Fez, the team of Pediatric Hematology-Oncology Service of the University Children Hospital of Rabat, the Embassy of Canada Morocco, the Avenir association, the Women, Values and Realizations Association, and the Terry Fox Foundation.

Thanks to the survivors who accepted to answer questions, and to their families.

\section{References}

[1] http://www.e-cancer.fr/cancerinfo/les-cancers/le-cancer-ch ez-l-enfant/complications-tardives-et-sequelles-eventuelles.

[2] http://www.cancer.gov/types/childhood-cancers/ccss.

[3] Frobisher, C., Lancashire, E. R., Winter, D. L., Jenkinson, H. C., and Hawkins, M. M. 2007. "Long-term Population-based Marriage Rates among Adult Survivors of Childhood Cancer in Britain.” Int. J. Cancer 121: 846-55.

[4] Ford, J. S., Kawashima, T., Whitton, J., Leisenring, W., Laverdière, C., Stovall, M., Zeltzer, L., Robison, L. L., and Sklar, C. A. 2014. "Psychosexual Functioning among Adult Female Survivors of Childhood Cancer: a Report from the Childhood Cancer Survivor Study." J. Clin. Oncol. 32 (28): 3126-36. doi: 10.1200/JCO.2013.54.1086. Epub 2014 Aug 11.

[5] van Dijk, E. M., van Dulmen-den Broeder, E., Kaspers, G. J., van Dam, E. W., Braam, K. I., and Huisman, J. 2008. "Psychosexual Functioning of Childhood Cancer Survivors." Psychooncology. 17 (5): 506-11.

[6] Apajasalo, M., Sintonen, H., Siimes, M. A., Hovi, L., Holmberg, C., and Boyd, H. 1996, et al. "Health-related Quality of Life of Adults Surviving Malignancies in Childhood." Eur. J. Cancer 32A (8): 1354-8.

[7] Feeny, D., Furlong, W., Mulhern, R. K., Barr, R. D., and Hudson, M. 1999. "A Framework for Assessing Health-related Quality of Life among Children with Cancer." Int. J. Cancer (12): 2-9.

[8] Cherlin, A. J. 2005. "American Marriage in the Early Twenty-first Century." Future Child 15: 33-55.

[9] Clare, F., Emma, R., Lancashire, David, L., Winter, Helen, C., Jenkinson, and Michael, M. Hawkins 2007. "Long-term Population-based Marriage Rates among Adult Survivors of Childhood Cancer in Britain." 
International Journal of Cancer 121 (4): 846-55.

[10] Rauck, A. M., Green, D. M., Yasui, Y., Mertens, A., and Robison, L. L. 1999. "Marriage in the Survivors of Childhood Cancer: a Preliminary Description from the Childhood Cancer Survivor Study." Med. Pediatr. Oncol. 33 (1): 60-3.

[11] Msefer Alaoui, F., and Benaicha, N. Jeunes marocains guéris d'un cancer de l'enfance, Que Deviennent-ils? Doctinews: 32-6.

[12] Msefer Alaoui, F., and Benaicha, N. 2015. "Moroccan Childhood Cancer Survivors: A Medical and Demographic Survey." Eposter SIOP.

[13] Haut-Commissariat au Plan, Recensement de la Population, Maroc, 2014. 